Conclusion This study shows that exposure to lead and cadmium may affect semen profile in male welding workers. Further studies are need to control for other potential confounding factors, and environmental assessment should be done to asses exposure pathways and concentration.

\section{$1727 f$ KNOWLEDGE ABOUT ADVERSE EFFECTS OF WORKING CONDITIONS MUST BE USED IN COUNSELLING BEFORE CONCEPTION AND DURING EARLY PREGNANCY}

1,2T Brand. 'Netherlands Centre for Occupational Diseases, Coronel Institute of Occupational Health, Academic Medical Centre, Amsterdam, The Netherlands; ${ }^{2}$ Arbo Unie, The Netherlands

\subsection{6/oemed-2018-ICOHabstracts.1237}

To find and use scientific evidence about the influence of working conditions on reproductive outcomes for practical use in a guideline for occupational and a website for pregnant women, parents to be, health care professionals and policy makers. We searched the literature for evidence concerning the effects of working conditions before, and during pregnancy on pregnancy outcome and development during early childhood. The working conditions with effects on pregnancy outcome were summarised in six groups: physically or mentally heavy work, shift work, chemical substances, physical factors (like noise) and infections. We looked at the following pregnancy outcomes spontaneous abortions, preterm birth, low birth weight, hypertension and congenital malformations and learning problems at early school age. Use of evidence: A practical guideline ${ }^{1}$ was made for occupational physicians in The Netherlands (also translated in English). It can be used by physicians in advising pregnant women and women breastfeeding their child. In another report 'Preconception care: a good beginning, ${ }^{2}$ the chapter about working conditions underlines the effect of exposure before conception on the embryo and fetus. In the third place the evidence was used on web$\operatorname{sites}^{3,4}$ for parents to be or pregnant women and can be used for preventive measures regarding working conditions preconceptionally or during pregnancy. There is growing evidence that working conditions before and during pregnancy can increase the number and severity of pregnancy complications. There is also evidence that early intervention can prevent this increase of pregnancy complications. The available evidence has been used for a guideline for occupational physicians and on a website for parents to be in order to take preventive measures. A summary of the evidence will be presented together with examples how to use the evidence in counselling before conception and during early pregnancy.

\section{$1727 \mathrm{~g}$ INCREASED RISKS OF CHILDHOOD CANCER AND INFANT DEATH IN THE OFFSPRING OF ELECTRONIC WORKERS}

${ }^{1} \mathrm{CC}$ Lin*, 1,2,3PC Chen. ${ }^{1}$ Institute of Occupational Medicine and Industrial Hygiene, National Taiwan University College of Public Health, Taipei, Taiwan; ${ }^{2}$ Department of Public Health, National Taiwan University College of Public Health, Taipei, Taiwan; ${ }^{3}$ Department of Environmental and Occupational Medicine, National Taiwan University Hospital and National Taiwan University College of Medicine, Taipei, Taiwan
In 1994, a hazardous waste site, polluted by the dumping of solvents from a former factory, was discovered in Taoyuan, Taiwan. The factory was built in 1970, and was in operation for the manufacturing of electronic appliances up until 1992. The objective of the study was to investigate the risks of childhood cancer and infant death in the offspring of electronic workers. We linked the databases of labour insurance, birth registration, and national cancer registry, which identified 40647 female workers ever employed in this factory who gave 40647 first live born singletons, and 47 of them developed cancers during 1979-2001. We also linked the databases of labour insurance, birth registration, and national death registry, identified 7202 male workers ever employed in this factory with 13592 live born children and 81 deaths in the first year after, excluding 861 children with potential maternal exposure from the same workplace.

We demonstrated evidence on the hypotheses that maternal exposure to organic solvents near conception increases the risk of cancer in offspring and that paternal exposure during preconception relates to infant mortality and deaths due to congenital malformation.

\section{Respiratory Disorders}

\section{A CROSS-SECTIONAL STUDY TO VALIDATE A SCREENING QUESTIONNAIRE TO DETERMINE PREVALENCE OF WORK-RELATED ASTHMA IN PRIMARY HEALTH CARE IN CHILE}

1,20 Solar*, ${ }^{1} \mathrm{G}$ Contreras, ${ }^{3} \mathrm{R}$ Sepulveda, ${ }^{4} \mathrm{~S}$ Castillo. ${ }^{1}$ School of Public Health, Faculty of Medicine, University of Chile, Santiago, Chile; ${ }^{2}$ Work, Employment and Health Inequities Faculty of Social Science of; (FLACSO), Chile; ${ }^{3}$ Primary Health Care Division, Ministry of Health, Santiago Chile; ${ }^{4}$ Primary Family Health Centre Dr R Yazigi, Lo Prado Municipality, Santiago, Chile

\subsection{6/oemed-2018-ICOHabstracts. 1239}

Introduction The attributable population risk of work-related asthma is between $10 \%$ and $20 \%$ of total cases of asthma. In Chile, the prevalence of common asthma is $10.2 \%$ but the proportion of occupational asthma (OA) is hidden and invisible. The study objective was to determine the prevalence of OA among asthmatic patients in regular control in the Primary Health Care (PHC) adult respiratory diseases program in Santiago, Chile.

Methods A descriptive, cross-sectional multicenter study of patients aged 20 to 64 years with diagnosis of asthma, according to medical records and functional respiratory tests; who were working or had worked and were in control in PHC in a healthcare district of Santiago. An Occupational Asthma Screening Questionnaire (OAS) was developed and validated in four PHC centres to identify occupational asthma in the population in control for bronchial asthma. The screening instrument included six questions with a scale from 6 to 16 points. The instrument was prepared with a panel of experts with validation of feasibility, reliability, logic, content, construct and criteria, using confirmatory diagnostic tests and blind medical evaluation by two specialists in respiratory diseases and occupational medicine as the gold standard. Patients were classified into three categories: OA, work-exacerbated asthma WEA or common asthma (CA), according to OAS and the medical evaluation. 
Result Two hundred and two patient completed the OAS and were evaluated by specialists. A cut-off of $>10.5$, points in the OAS yielded sensitivity of 0.79 ; specificity of 0.78 ; VP (+) 0.22 and VP (-) 0.98 . The detected prevalence of OA was $8.2 \%$ and asthma exacerbated by work was $14.2 \%$, for a total prevalence of work-related asthma of $22 \%$.

Discussion The findings support implementation of an occupational asthma surveillance program in the PHC network in Santiago, Chile to benefit workers and to improve the results of PHC respiratory health programs.

\section{THE ROLE AND MECHANISM OF EMT IN MARCO- MEDIATED SILICOSIS IN RATS}

${ }^{1}$ Sanqiao Yao, ${ }^{2}$ Meng Yang, ${ }^{1} \mathrm{Na}$ Wang, ${ }^{2}$ Yuping Bai, 'Xinlai Qian, ${ }^{1}$ Weidong Wu, 'Zhen An, 'Guanghui Zhang, 'Haibin Li. 'Xinxiang Medical University, Xinxiang, China; ${ }^{2}$ North China University of Science and Technology, Tangshan, China

\subsection{6/oemed-2018-ICOHabstracts. 1240}

Objectives The animal model of silicosis of non-exposed tracheal instillation induced by $\mathrm{SiO}_{2}$ in rats lung fibrosis were studied. To investigate:

1. whether $\mathrm{SiO}_{2}$ can induce fibrosis in lung tissue by induction of epithelial interstitial transformation (EMT);

2. EMT in poly guanine nucleotide (PolyG) molecular biology mechanism of silicosis fibrosis reversal.

3. the effects of PolyG preventive and therapeutic interventions on EMT in rats exposed to silica dust and the effect of intervention at different time points.

Methods Ninety-six healthy male Sprague-Dawley (SD) rats weighing $180 \sim 220 \mathrm{~g}$ were randomly divided into normal saline group $(n=32)$, silicosis model group $(n=32)$, PolyG prevention group $(n=16)$ and PolyG (28 days) treatment group $(n=16)$. After the rats were anaesthetised with ether, the control group was injected with $1 \mathrm{ml}$ normal saline by bronchial instillation. The rats in other groups were treated with nonexposed tracheal infusion of a one-time infusion of $50 \mathrm{ml} / \mathrm{L}$ silica suspension $1 \mathrm{ml}$. The rats in the PolyG preventive group were treated with PolyG $2.5 \mathrm{mg} / \mathrm{kg}$ body weight (the weight of each rat was determined 28 days after modelling) at the same time by tail vein injection. The rats in the PolyG treatment group were treated with intravenous injection of PolyG $2.5 \mathrm{mg} / \mathrm{Kg}$ body weight (by modelling 28 days after each rat weight to determine the appropriate dose). The rats in the polyG prevention group and the treatment group were sacrificed on the 28th day and the 56th day after the corresponding administration. Silicosis model group and saline control group also have 8 rats were sacrificed at the same time point. (Macrophage receptor with collagenous structure, MARCO), E-cadherin (E-cadherin) were detected by Western blotting. The macrophage receptor (MARCO) Cadherin, E-cadherin, $\alpha$ smooth muscle actin ( $\alpha$-SMA), vimentin and type I and type III collagen; the right amount of lung tissue, The relative expression levels of E-cadherin, $\alpha$-SMA, vimentin and type I and type III procollagen mRNA were detected by Real-time PCR. The right middle lobe tissue was fixed with 4\% paraformaldehyde, paraffin section, the histopathological changes of lung tissue were observed by $\mathrm{HE}$ staining and Masson staining. Immunohistochemical staining was used to detect the localization and expression of E-cadherin, $\alpha$-SMA and vimentin in lung tissue.

\section{Results}

1. Histopathological changes of lung tissue in different groups: The lung tissue structure of the rats in the control group was normal, and the infiltration of inflammatory cells was observed in the surrounding area, and the lung tissue structure of the control group did not change significantly over time; There were a large number of inflammatory cell infiltration in the lung tissue of the rats in the silicosis model, and the typical fibrous nodules were formed. The nodules were composed of macrophages and fibroblasts. Some areas of the alveolar structure still existed, showing different alveolar walls and small blood vessel wall thickening; with the dying time, the number of fibrous silicon nodules increased, some of the silicon nodules have a trend of integration and becoming bigger, in particular, is completely fibrous tissue, or even fibrotic changes; In PolyG prevention group and treatment group, the number of cell nodules or silicon nodules was lower than that of the model group; the degree of lesion in the rats was significantly lower than that in the control group. The results of immunohistochemistry showed that $\alpha$-SMA and vimentin positive cells were significantly increased in the silicosis model group, and the number of Ecadherin positive cells was significantly decreased. After administration of PolyG, the number of E-cadherin-positive cells and the number of $\alpha$-SMA and vimentin-positive cells were significantly increased.

2. The expression of MARCO, E-cadherin, $\alpha$-SMA and vimentin in the lung tissue of the control group were not significantly different $(p<0.05)$. There was no significant difference in the expression of MARCO and EMT-related proteins in rat lung tissue at different time points $(\mathrm{p}<0.05)$. The levels of Ecadherin in the lung tissue of the silicosis model group were increased with the prolongation of observation time $(\mathrm{p}<0.05)$. The expression levels of MARCO, $\alpha$-SMA and vimentin protein in the lung tissue of the PolyG intervention group (prophylactic and therapeutic) were lower than those in the control group, but the E-cadherin was higher than that of the silicosis group model group $(\mathrm{p}<0.05)$.

3. The comparison of mRNA expression levels of E-cadherin, $\alpha$ SMA and vimentin in lung tissue of different groups: the expression of E-cadherin, $\alpha$-SMA and vimentin mRNA in lung tissue of all groups were the same as those of corresponding protein.

4. The expression of collagen I and III in the lung tissue of different groups were significantly higher than those in the control group $(\mathrm{p}<0.05)$. There was no significant difference in the expression of collagen I and III in rat lung tissue of control groups at different time points $(\mathrm{p}<0.05)$. The expression level of collagen I and III in the lung tissue of silicosis model group increased with the prolongation of dying time $(\mathrm{p}<0.05)$. The protein content of collagen I and III in PolyG intervention group was significantly higher than that in the control group $(\mathrm{p}<0.05)$. The mRNA expression of type I and III procollagen were the same as that of I and III collagen.

\section{Conclusions}

- The protein content and mRNA expression of E-cadherin were decreased, while $\alpha-S M A$ and vimentin in silicosis model group were increased, which indicated that there was epithelial-mesenchymal transition in the development of silicosis.

- After inhibition of MARCO combined with $\mathrm{SiO}_{2}$, the protein content and mRNA expression of $\alpha-S M A$ and vimentin were 